\title{
Levantamento taxonômico e caracterização do hábitat de insetos aquáticos em Unidades de Conservação de uma ilha subtropical
}

\author{
Jéssica da Rosa Pires ${ }^{1 *}$ \\ Leonardo Kleba Lisboa ${ }^{2}$ \\ Aurea Luiza Lemes Da Silva ${ }^{1}$ \\ Mauricio Mello Petrucio ${ }^{1}$ \\ Ana Emilia Siegloch ${ }^{3}$ \\ ${ }^{1}$ Laboratório de Ecologia de Águas Continentais, Centro de Ciências Biológicas \\ Departamento de Ecologia e Zoologia, Universidade Federal de Santa Catarina \\ Campus Universitário Reitor João David Ferreira Lima, CEP 88010-970, Florianópolis - SC, Brasil \\ ${ }^{2}$ Universidade Estadual do Rio de Janeiro, Rio de Janeiro - RJ, Brasil \\ ${ }^{3}$ Universidade do Planalto Catarinense, Lages - SC, Brasil \\ * Autor para correspondência \\ jessica.rosap@hotmail.com
}

Submetido em 03/09/2014

Aceito para publicação em 09/06/2015

\section{Resumo}

Inventários taxonômicos são a base de diversos estudos ecológicos e possibilitam conhecer melhor a biodiversidade local e regional. O objetivo deste trabalho foi inventariar a fauna de insetos aquáticos de uma ilha subtropical, bem como gerar informações sobre os hábitats utilizados pelos táxons encontrados. Foram selecionadas duas regiões em bom estado de conservação na Ilha de Santa Catarina, estado de Santa Catarina (SC), o Parque Municipal da Lagoa do Peri e a Unidade de Conservação Ambiental Desterro. Os invertebrados aquáticos foram coletados com amostrador de Surber (em ambiente lótico) e draga de Eckman-Birge (ambiente lêntico) entre 2009 e 2012. Foram encontrados 60 táxons compreendidos em oito ordens taxonômicas. Com isso, foram realizados 19 novos registros de famílias de insetos aquáticos para SC. Nos locais deste estudo não foram observadas 13 das famílias já conhecidas para SC, conforme comparativo com artigos publicados até julho/2014. Quanto ao hábitat, a riqueza diferiu entre os tipos de habitats amostrados, com riqueza menor no substrato "areia". O estudo representa uma importante contribuição para o conhecimento de insetos aquáticos no estado de Santa Catarina, especialmente para a biodiversidade de ilhas.

Palavras-chaves: Ambiente aquático; Biodiversidade aquática; Inventário faunístico; Santa Catarina; Substrato

\section{Abstract}

Taxonomic survey and characterization of the habitat of aquatic insects in protected areas in a subtropical island. Taxonomic inventories are the basis of several ecological studies and they enable a better understanding of the local and regional biodiversity. This paper aimed to survey the aquatic insect fauna in a subtropical island, as well as to generate information on the habitats used by the taxa found. Two regions 
showing a good state of environmental conservation in the Santa Catarina Island, in Santa Catarina, Brazil, were selected: "Lagoa do Peri" Municipal Park and "Desterro" Environmental Protected Area. Aquatic invertebrates were collected by using a Surber sampler (in a lotic environment) and an Eckman-Birge dredger (in a lentic environment) between 2009 and 2012. Sixty taxa were found, belonging to eight taxonomic orders. Thus, there were 19 new registers of aquatic insect families for Santa Catarina. At the sites of this study, 13 families already known for Santa Catarina were not observed, according to a comparison with articles published until July 2014. As for the habitat, richness differed between the types of the habitats sampled, with lower richness in the substrate "sand". The study represents a significant contribution to knowledge on aquatic insects in Santa Catarina, especially regarding the biodiversity in islands.

Keys words: Aquatic biodiversity; Aquatic environment; Faunal inventory; Santa Catarina; Substrate

\section{Introdução}

Insetos aquáticos são fundamentais na ciclagem de nutrientes e no fluxo de energia dos ambientes aquáticos e apresentam alta plasticidade ambiental (TRIVINHO-STRIXINO; STRIXINO, 1993). Esses organismos podem passar parte de sua vida (estágios imaturos) ou todo o ciclo no leito de ambientes aquáticos, associados ao substrato inorgânico (matacão, seixos e cascalhos), orgânico (folhas, galhos, troncos) ou enterrados no sedimento. Eles respondem às perturbações ambientais de forma distinta, apresentando tanto espécies sensíveis como tolerantes à poluição, sendo assim bons bioindicadores da qualidade ambiental (GALDEAN et al., 2000; CALLISTO et al., 2001).

Os invertebrados aquáticos têm sua distribuição controlada por vários fatores: disponibilidade e qualidade de recursos alimentares, tipo de sedimento, substrato, temperatura do meio, concentração de oxigênio dissolvido, condutividade, velocidade da água e pH (TUNDISI; MATSUMURA-TUNDISI, 2008). Dentre estes, a composição do substrato destaca-se por ser essencial na estruturação da comunidade aquática (BEISEL et al., 1998). Este recurso oferece abrigo contra os predadores, os protege da força do fluxo da água e contribui para maior disponibilidade de alimento através do acúmulo de material orgânico autóctone e alóctone (KIKUCHI; UIEDA, 1998; OLIVEIRA; CALLISTO, 2010). No geral, ambientes com sedimento fino, como areia, apresentam menor riqueza e diversidade. Isto porque apresentam menor disponibilidade de recurso alimentar e menor estabilidade em corredeiras, pois são facilmente carreados pela água (VUORI; JOENSUU, 1996; KALLER; HARTMAN, 2004).
As comunidades de insetos aquáticos na região sul do Brasil são conhecidas principalmente através de biomonitoramento e estudos ecológicos. Entretanto, no estado de Santa Catarina estes trabalhos ainda são escassos. Muito da entomofauna catarinense foi pesquisada e descrita por Fritz Plaumann (RAIMUNDI et al., 2013), um entomólogo alemão que coletou extensivamente exemplares de insetos no estado e criou uma vasta coleção entomológica. Outro grande entusiasta da entomologia catarinense foi o naturalista também alemão Fritz Müller, que descreveu, além de muitos outros táxons, várias espécies de insetos aquáticos. Fritz Müller teve como maior interesse as descrições morfológicas de larvas e pupas das ordens Trichoptera e Diptera (FROEHLICH, 1966).

Nos atuais trabalhos de cunho científico sobre biodiversidade aquática em Santa Catarina, destacamse os estudos com ênfase no levantamento da família Leptophlebiidae (RAIMUNDI et al., 2013) e, bem como, descrições de novas espécies por: Paprocki et al. (2004), Alves et al. (2006), Salles et al. (2004), Sterz et al. (2011) e Novaes e Bispo (2014). Considerando os estudos levantados, foi registrado um total de 52 famílias de insetos aquáticos para o estado, sendo este número distribuído entre as ordens Diptera (12 famílias), Trichoptera (12), Coleoptera (8), Odonata (7), Ephemeroptera (6), Hemiptera (3), Plecoptera (2), Lepidoptera (1) e Megaloptera (1).

Desse modo, estudos com enfoque em inventário taxonômico são relevantes para se conhecer melhor a biodiversidade de insetos aquáticos no estado, especialmente na Ilha de Santa Catarina onde as áreas de vegetação natural sofrem grande pressão por parte 
das atividades antrópicas. Atualmente, os fragmentos de mata remanescentes encontram-se principalmente em Unidades de Conservação ou no alto dos morros, constituídos por vegetação secundária em diferentes estágios de sucessão e regeneração (BISHEIMER et al., 2013). O objetivo deste trabalho foi inventariar a fauna de insetos aquáticos na Ilha de Santa Catarina com base em estágios imaturos, comparando com outros inventários realizados no estado catarinense, além de gerar informações sobre os hábitats utilizados pelos táxons encontrados.

\section{Material e Métodos}

\section{Área de estudo}

$\mathrm{O}$ estudo foi realizado em duas áreas em bom estado de conservação da Ilha de Santa Catarina, SC. Estas áreas estão compreendidas pelo Parque Municipal da Lagoa do Peri (PMLP), na porção sul da ilha, e pela Unidade de Conservação Ambiental Desterro (UCAD), na porção norte da ilha (Figura 1). Segundo Caruso (1990), a Ilha de Santa Catarina foi muito explorada para o estabelecimento da agricultura e, em decorrência disto, atualmente não apresenta matas primárias propriamente ditas. O clima da região é classificado como CFa segundo Köppen com verões quentes e chuvas bem distribuídas ao longo do ano (HENNEMANN; PETRUCIO, 2011). Porém os registros pluviométricos históricos (desde 1992) mostram taxas mais elevadas de precipitação nos meses mais quentes (entre dezembro e março) (LISBOA et al., 2015).

A Lagoa do Peri $\left(27^{\circ} 44^{\prime} \mathrm{S}\right.$ e $\left.48^{\circ} 31^{\prime} \mathrm{W}\right)$ está inserida em uma área de preservação de Floresta Ombrófila Densa de 20,1 $\mathrm{km}^{2}$.É o maior manancial de água doce da Ilha de Santa Catarina e apresenta um espelho d'água de $5,7 \mathrm{~km}^{2}$ rodeado por morros cobertos por Mata Atlântica e uma restinga típica de vegetação litorânea, que está 3 $\mathrm{m}$ acima do nível do mar separando a lagoa do mesmo (LISBOA et al., 2011). A Lagoa do Peri apresenta uma profundidade máxima de $11 \mathrm{~m}$ na sua porção central e profundidade média de $7 \mathrm{~m}$ (OLIVEIRA, 2002). A bacia hidrográfica drena uma área de $20,1 \mathrm{~km}^{2}$ e é composta por dois principais afluentes de $3^{\mathrm{a}}$ ordem, Cachoeira
Grande e Ribeirão Grande. O canal de extravasamento das águas da lagoa em direção ao mar (canal Sangrador) é de sentido único (HENNEMANN; PETRUCIO, 2011).

FIGURA 1: Localização da UCAD, região noroeste, e do Parque Municipal da Lagoa do Peri, região sudeste, na Ilha de Santa Catarina, SC.

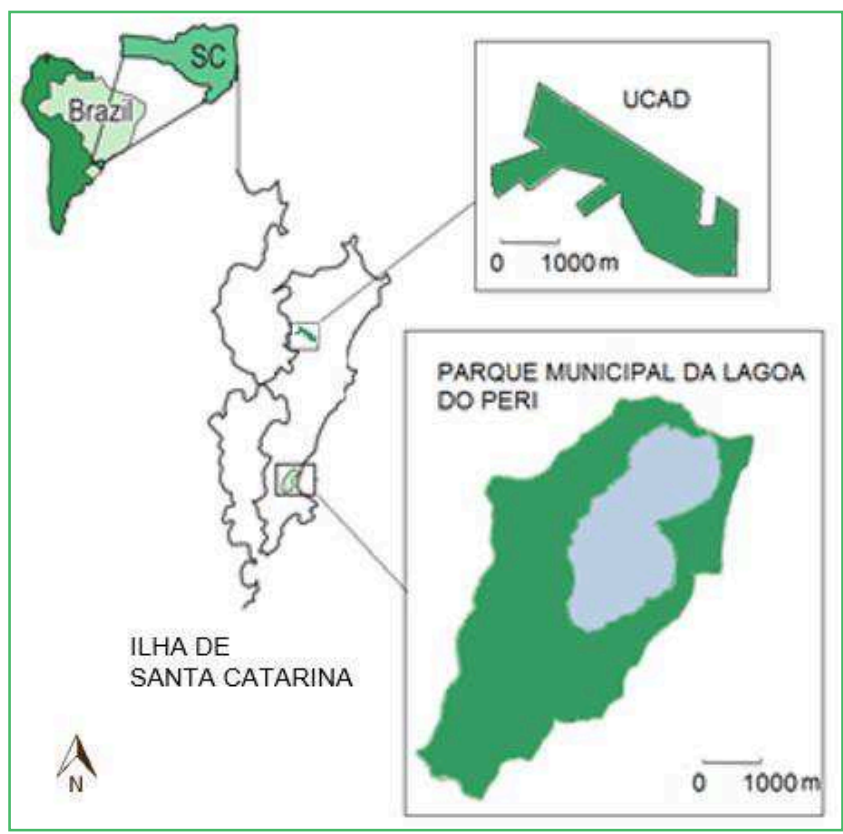

A UCAD $\left(27^{\circ} 31^{\prime} 50,8\right.$ 'S e $48^{\circ} 30^{\prime} 44,3$ 'O) compreende uma área de $4,9 \mathrm{~km}^{2}$ sendo uma área natural protegida pela Universidade Federal de Santa Catarina. Sua vegetação é composta por Floresta Ombrófila Densa em quatro estádios de regeneração: capoeirinha (com até seis anos de desenvolvimento), capoeira (aproximadamente 20 anos), capoeirão (vegetação se desenvolvendo por um período de aproximadamente 30 anos) e floresta secundária (área apenas explorada seletivamente) (BONNET; QUEIROZ, 2006). Alguns riachos da Unidade de Conservação fazem parte da bacia hidrográfica do Rio Ratones, importante recurso hídrico da região norte da ilha, outros riachos pertencem a bacia do Saco Grande.

\section{Amostragem}

As coletas foram realizadas entre 2009 e 2012 abrangendo ambientes lóticos e um ambiente lêntico. Nos ambientes lóticos, amostras dos substratos (folhas, pedras e areia em trechos de corredeira e remanso) 
foram coletadas com auxílio de amostrador de "Surber" com área amostral de $0,0361 \mathrm{~m}^{2}$ e tamanho de malha de $250 \mu \mathrm{m}$. No PMLP, foram amostrados 11 riachos e na UCAD cinco riachos em distintos estados de conservação da mata ripária. $\mathrm{O}$ ambiente lêntico foi representado pela Lagoa do Peri, cujas, coletas foram realizadas no período de março 2008 a junho de 2009 com draga de Eckman-Birge $\left(0,0625 \mathrm{~m}^{2}\right)$, em cinco áreas que melhor representam os diferentes biótopos da lagoa, totalizando 16 amostras. No total foram 105 pontos de amostragem no ambiente lótico (32 em folha/ remanso, 22 em pedra/remanso, 20 em folha/corredeira, 17 em pedra/corredeira, nove em areia/remanso e $11 \mathrm{sem}$ definição de hábitat).

Depois das coletas, as amostras foram levadas ao laboratório de Ecologia de Águas Continentais, lavadas sob peneira de malha $250 \mu \mathrm{m}$, triadas com estereomicroscópio e fixadas em álcool 70\%. Para identificação dos insetos aquáticos no nível taxonômico de família foi utilizada bibliografia especializada (DOMÍNGUEZ; FERNÁNDEZ, 2009; MUGNAI et al., 2010) e auxílio de especialistas.

\section{Análise de dados}

A riqueza total de táxons e a eficiência do esforço de amostragem foram avaliadas por curvas de acumulação randomizadas e por estimadores não paramétricos de riqueza (Chao 1, 2, Bootstrap e Jackknife 1 e 2). Curvas randomizadas foram usadas para evitar variação na forma da curva durante a adição das amostras (COLWELL; CODDINGTON, 1994). As curvas de acumulação foram geradas separadamente para o ambiente lêntico e lótico a partir de 500 aleatorizações no software EstimateS ${ }^{\circledR}$.

A riqueza de espécies é dependente do esforço de amostragem, portanto, pode-se padronizar o esforço em termos de área ou em número de indivíduos através da rarefação (um tipo de interpolação, GOTELLI; COLWELL, 2001). Neste estudo, o esforço de coleta foi diferente entre os tipos de hábitats amostrados no sistema lótico, assim para viabilizar a comparação de riqueza entre os hábitats foi usado o método da rarefação. As curvas foram geradas com base em 1.000 aleatorizações pelo programa Past (HAMMER et al., 2001).
Para comparação com a entomofauna aquática do estado catarinense foi realizada uma busca na base de dados Scielo e Scopus. A pesquisa foi realizada utilizando combinações entre as seguintes palavras-chaves: inventário, insetos aquáticos, Santa Catarina, macroinvertebrados bentônicos, aquatic macroinvertebrates, aquatic insect, aquatic invertebrates bem como cada ordem de inseto aquático (Collembola, Coleoptera, Diptera, Ephemeroptera, Hemiptera, Lepidoptera, Megaloptera, Odonata, Plecoptera e Trichoptera). A busca por trabalhos publicados ocorreu até julho de 2014. Nesta revisão foram descartadas descrições taxonômicas publicadas ou registros em museus e coleções científicas, pois estes podem gerar uma grande quantidade isolada de registros de dados, portanto, um potencial conflito em famílias com gêneros e espécies aquáticas e terrestres. Treze artigos constatando a ocorrência de famílias de insetos aquáticos foram encontrados.

\section{Resultados}

Neste estudo, foram registradas 60 famílias e oito ordens taxonômicas de insetos aquáticos. Das ordens de insetos aquáticos já registradas para Santa Catarina, Megaloptera e as seguintes famílias não ocorreram na ilha: Lestidae e Corduliidae (Odonata); Athericidae, Blepharicidae, Ephydridae e Rhagionidae (Diptera); Trichorythidae (Ephemeroptera); Scarabaeidae e Carabidae (Coleoptera) e Naucoridae e Corixidae (Hemiptera. A Tabela 1 apresenta os resultados do levantamento dos insetos aquáticos encontrados no estado de Santa Catarina com base na busca dos artigos científicos. Ao comparar a composição faunística encontrada nestes estudos com o levantamento feito na Ilha de Santa Catarina (Tabela 2) foi possível verificar 19 novos registros de famílias de insetos aquáticos para o estado: Corethrellidae, Chaoboridae, Dixidae, Psychodidae, Euthyplociidae, Polymitarcidae, Noteridae, Dysticidae, Scistidae, Lutrochidae, Belostomatidae, Macrovellidae, Mesovellidae, Vellidae, Megapodagrionidae, Perelestidae, Dicteriadidae, Sericostomatidae e Calamoceratidae. 
TABELA 1: Relação dos artigos selecionados nas bases de dados para Santa Catarina.

\begin{tabular}{|c|c|c|c|}
\hline Artigos & Assunto & Área & Riqueza* \\
\hline Paprocki et al. (2004) & Check-list da ordem & Trichoptera/Brasil & 11 \\
\hline Salles et al. (2004) & Check-list da ordem & Ephemeroptera/Brasil & 5 \\
\hline Hepp et al. (2005) & Biomonitoramento & Ribeirão Garcia, Blumenau/SC & 2 \\
\hline Rodrigues et al. (2005) & Biomonitoramento & Rio Mãe Luzia, Treviso/SC & 33 \\
\hline Alves et al. (2006) & Associação com macrófitas & Baixada do Maciambu, Palhoça/SC & 9 \\
\hline Molozzi et al. (2007) & Biomonitoramento & Bacia do Itajaí, Gaspar e Agrolândia/SC & 32 \\
\hline Dias et al. (2009) & Biomonitoramento & Bacia do Itajaí, Gaspar e Agrolândia/SC & 29 \\
\hline Pinto e Lamas (2010) & Nova espécie / Odonata & São Bento do Sul/SC & 1 \\
\hline Battistoni et al. (2010) & Levantamento & Rio Jacuntinga, Concórdia/SC & 5 \\
\hline Disner et al. (2011) & Biomonitoramento & Rio Guamerim, São Miguel do Oeste/SC & 2 \\
\hline Raimundi et al. (2013) & $\begin{array}{l}\text { Levantamento / Leptophlebiidae } \\
\text { (Ephemeroptera) }\end{array}$ & Oeste de SC & 1 \\
\hline Novaes e Bispo (2014) & Nova espécie / Plecoptera & $\mathrm{SC}$ & 1 \\
\hline Mendes e Pinho (2014) & Check-list da família & Chironomidae/Brasil & 1 \\
\hline
\end{tabular}

*Riqueza em nível de família de insetos aquáticos

TABELA 2: Composição taxonômica de insetos aquáticos na Ilha de Santa Catarina no período de 2009 a 2012 e no estado de Santa Catarina segundo artigos científicos publicados até junho de 2014.

\begin{tabular}{|c|c|c|c|c|c|c|c|c|c|c|}
\hline \multirow{4}{*}{ Táxons } & \multicolumn{9}{|c|}{ Ilha de Santa Catarina } & \multirow[t]{4}{*}{ SC } \\
\hline & \multicolumn{8}{|c|}{ Lótico } & Lêntico & \\
\hline & \multicolumn{6}{|c|}{ PMLP } & \multicolumn{2}{|c|}{ UCAD } & PMLP & \\
\hline & FR & PR & FC & PC & AR & FAR & FR & PR & AR & \\
\hline Collembola & $\mathrm{x}$ & & & & $\mathrm{x}$ & & $\mathrm{x}$ & $\mathrm{x}$ & & $\mathrm{x}$ \\
\hline \multicolumn{11}{|l|}{ Colepotera } \\
\hline Carabidae & & & & & & & & & & $\mathrm{x}$ \\
\hline Curculionidae & $\mathrm{x}$ & & $\mathrm{x}$ & $\mathrm{x}$ & & & & & & $\mathrm{x}$ \\
\hline Dryopidae & $\mathrm{x}$ & & $\mathrm{x}$ & & & & & & & $\mathrm{x}$ \\
\hline Dytiscidae & $\mathrm{x}$ & $\mathrm{x}$ & $\mathrm{x}$ & $\mathrm{x}$ & $\mathrm{x}$ & & & $\mathrm{x}$ & $\mathrm{x}$ & \\
\hline Elmidae & $\mathrm{x}$ & $\mathrm{x}$ & $\mathrm{x}$ & $\mathrm{x}$ & & & $\mathrm{x}$ & $\mathrm{x}$ & $\mathrm{x}$ & $\mathrm{x}$ \\
\hline Gyrinidae & $\mathrm{x}$ & $\mathrm{x}$ & $\mathrm{x}$ & $\mathrm{x}$ & $\mathrm{x}$ & & & $\mathrm{x}$ & $\mathrm{x}$ & $\mathrm{x}$ \\
\hline Hydrophilidae & & & & & & & $\mathrm{x}$ & & & $\mathrm{x}$ \\
\hline Lutrochidae & $\mathrm{x}$ & & $\mathrm{x}$ & & & & $\mathrm{x}$ & & & \\
\hline Naucoridae & & & & & & & & & & $\mathrm{x}$ \\
\hline Noteridae & & $\mathrm{x}$ & $\mathrm{x}$ & & & & & & & \\
\hline Psephenidae & $\mathrm{x}$ & $\mathrm{x}$ & $\mathrm{x}$ & $\mathrm{x}$ & & & $\mathrm{x}$ & $\mathrm{x}$ & & $\mathrm{x}$ \\
\hline Scarabaeidae & & & & & & & & & & $\mathrm{x}$ \\
\hline Scirtidae & & & $\mathrm{x}$ & $\mathrm{x}$ & & & & & & \\
\hline Staphylinidae & $\mathrm{x}$ & & $\mathrm{x}$ & & $\mathrm{x}$ & & $\mathrm{x}$ & & & \\
\hline \multicolumn{11}{|l|}{ Diptera } \\
\hline Athericidae & & & & & & & & & & $\mathrm{x}$ \\
\hline Blepharicidae & & & & & & & & & & $\mathrm{x}$ \\
\hline Ceratopogonidae & $\mathrm{x}$ & $\mathrm{x}$ & $\mathrm{x}$ & $\mathrm{x}$ & & & $\mathrm{x}$ & $\mathrm{x}$ & $\mathrm{x}$ & $\mathrm{x}$ \\
\hline Chaoboridae & $\mathrm{x}$ & $\mathrm{x}$ & $\mathrm{x}$ & $\mathrm{x}$ & $\mathrm{x}$ & & & & $\mathrm{x}$ & \\
\hline Chironomidae & $\mathrm{x}$ & $\mathrm{x}$ & $\mathrm{x}$ & $\mathrm{x}$ & $\mathrm{x}$ & $\mathrm{x}$ & $\mathrm{x}$ & $\mathrm{x}$ & $\mathrm{x}$ & $\mathrm{x}$ \\
\hline
\end{tabular}




\begin{tabular}{|c|c|c|c|c|c|c|c|c|c|c|}
\hline Corethrellidae & $\mathrm{x}$ & & & & & & & & & \\
\hline Culicidae & & & & $\mathrm{x}$ & & & & & & $\mathrm{x}$ \\
\hline Dixidae & & $\mathrm{x}$ & $\mathrm{x}$ & & & & $\mathrm{x}$ & $\mathrm{x}$ & & \\
\hline Empididae & $\mathrm{x}$ & $\mathrm{x}$ & $\mathrm{x}$ & $\mathrm{x}$ & & & $\mathrm{x}$ & $\mathrm{x}$ & & $\mathrm{x}$ \\
\hline Ephydridae & & & & & & & & & & $\mathrm{x}$ \\
\hline Muscidae & $\mathrm{x}$ & & $\mathrm{x}$ & $\mathrm{x}$ & & & & $\mathrm{x}$ & & $\mathrm{x}$ \\
\hline Psychodidae & $\mathrm{x}$ & $\mathrm{x}$ & $\mathrm{x}$ & $\mathrm{x}$ & & & $\mathrm{x}$ & $\mathrm{x}$ & & \\
\hline Rhagionidae & & & & & & & & & & $\mathrm{x}$ \\
\hline Simuliidae & $\mathrm{x}$ & $\mathrm{x}$ & $\mathrm{x}$ & $\mathrm{x}$ & & & $\mathrm{x}$ & $\mathrm{x}$ & & $\mathrm{x}$ \\
\hline Stratiomydae & & $\mathrm{x}$ & $\mathrm{x}$ & $\mathrm{x}$ & & & & & & $\mathrm{x}$ \\
\hline Tabanidae & $\mathrm{x}$ & & $\mathrm{x}$ & $\mathrm{x}$ & & $\mathrm{x}$ & & & & $\mathrm{x}$ \\
\hline Tipulidae & $\mathrm{x}$ & $\mathrm{x}$ & $\mathrm{x}$ & $\mathrm{x}$ & $\mathrm{x}$ & & $\mathrm{x}$ & $\mathrm{x}$ & & $\mathrm{x}$ \\
\hline \multicolumn{11}{|l|}{ Ephemeroptera } \\
\hline Baetidae & $\mathrm{x}$ & $\mathrm{x}$ & $\mathrm{x}$ & $\mathrm{x}$ & $\mathrm{x}$ & & $\mathrm{x}$ & $\mathrm{x}$ & $\mathrm{x}$ & $\mathrm{x}$ \\
\hline Caenidae & $\mathrm{x}$ & & & & $\mathrm{x}$ & $\mathrm{x}$ & & & & $\mathrm{x}$ \\
\hline Euthyplociidae & $\mathrm{x}$ & $\mathrm{x}$ & $\mathrm{x}$ & $\mathrm{x}$ & & & & $\mathrm{x}$ & & \\
\hline Leptohyphidae & $\mathrm{X}$ & $\mathrm{x}$ & $\mathrm{x}$ & $\mathrm{x}$ & & & & & & $\mathrm{x}$ \\
\hline Leptophlebiidae & $\mathrm{x}$ & $\mathrm{x}$ & $\mathrm{x}$ & $\mathrm{x}$ & & & $\mathrm{x}$ & $\mathrm{x}$ & & $\mathrm{x}$ \\
\hline Melanemerellidae & & & & & & & & & & $\mathrm{x}$ \\
\hline Oligoneuriidae & & & & & & & & & & $\mathrm{x}$ \\
\hline Polymitarcidae & & $\mathrm{x}$ & & $\mathrm{x}$ & & & & & & \\
\hline Tricorythidae & & & & & & & & & & $\mathrm{x}$ \\
\hline
\end{tabular}

\section{Hemiptera}

Belostomatidae

$\mathrm{X}$

Corixidae

Gerridae

X $\quad \mathrm{x}$

Macroveliidae

Mesoveliidae

Veliidae

$\begin{array}{llll}\mathrm{x} & \mathrm{X} & & \mathrm{x} \\ \mathrm{X} & \mathrm{X} & \mathrm{x} & \mathrm{x}\end{array}$

Lepidoptera

Pyralidae

X $\quad \mathrm{X} \quad \mathrm{x}$

x $\quad \mathrm{x}$

\section{Megaloptera}

Corydalidae

\section{Odonata}

Aeshnidae

Calopterygidae

Coenagrionidae

Corduliidae

Dicteriadidae

Gomphidae

Lestidae

Libellulidae

Megapodagrionidae

Perelestidae

Protoneuridae $x$ $x$

$\mathrm{x}$ $\mathrm{x}$ $\mathrm{x}$ $\mathrm{X}$ $x$ $\mathrm{x}$ $x$

Plecoptera

Gripopterygidae

Perlidae

$\begin{array}{llll}x & x & x & x \\ x & x & x & x\end{array}$

$\begin{array}{lll} & \mathrm{x} & \mathrm{x} \\ \mathrm{x} & \mathrm{x} & \mathrm{x}\end{array}$

$\begin{array}{llll}X & X & X\end{array}$

$\mathrm{X} \quad \mathrm{X} \quad \mathrm{X} \quad \mathrm{X}$

X $\quad \mathrm{X} \quad \mathrm{X} \quad \mathrm{X}$

$\mathrm{X}$

X $\quad \mathrm{X}$

$\mathrm{X}$

$\mathrm{X}$

$\mathrm{X}$

$\mathrm{X}$

$\mathrm{X}$

$\mathrm{X}$

X X

$\mathrm{X}$

X $\quad \mathrm{X}$

$\mathrm{x} \quad \mathrm{x}$

$\begin{array}{llll}\mathrm{X} & \mathrm{X} & \mathrm{X}\end{array}$

$\mathrm{X}$

X $\quad \mathrm{X}$

X $\quad \mathrm{X} \quad \mathrm{X} \quad \mathrm{X}$

$\mathrm{x} \quad \mathrm{x} \quad \mathrm{x}$

$\mathrm{X}$

Trichoptera

Calamoceratidae

$\begin{array}{llll}X & X & X\end{array}$

$\mathrm{X} \quad \mathrm{X}$

X 


\begin{tabular}{|c|c|c|c|c|c|c|c|c|c|c|}
\hline Glossosomatidae & $\mathrm{x}$ & $\mathrm{x}$ & $\mathrm{x}$ & $\mathrm{x}$ & & & & & & $\mathrm{x}$ \\
\hline Helicopsychidae & $\mathrm{x}$ & $\mathrm{x}$ & $\mathrm{x}$ & $\mathrm{x}$ & & & & & & $\mathrm{x}$ \\
\hline Hydroptilidae & $\mathrm{x}$ & $\mathrm{x}$ & $\mathrm{x}$ & $\mathrm{x}$ & & & $\mathrm{x}$ & $\mathrm{x}$ & & $\mathrm{x}$ \\
\hline Hydrobiosidae & $\mathrm{x}$ & $\mathrm{x}$ & $\mathrm{x}$ & $\mathrm{x}$ & & & & $\mathrm{x}$ & & $\mathrm{x}$ \\
\hline Hydropsychidae & $\mathrm{x}$ & $\mathrm{x}$ & $\mathrm{x}$ & $\mathrm{x}$ & & & $\mathrm{x}$ & $\mathrm{x}$ & $\mathrm{x}$ & $\mathrm{x}$ \\
\hline Lepdostomatidae & & & & & & & & & & $\mathrm{x}$ \\
\hline Leptoceridae & $\mathrm{x}$ & $\mathrm{x}$ & $\mathrm{x}$ & $\mathrm{x}$ & & & $\mathrm{x}$ & $\mathrm{x}$ & $\mathrm{x}$ & $\mathrm{x}$ \\
\hline Limnephilidae & & & & & & & $\mathrm{x}$ & $\mathrm{x}$ & & \\
\hline Odontoceridae & $\mathrm{x}$ & & & $\mathrm{x}$ & & & $\mathrm{x}$ & $\mathrm{x}$ & $\mathrm{x}$ & $\mathrm{x}$ \\
\hline Philopotamidae & $\mathrm{x}$ & $\mathrm{x}$ & $\mathrm{x}$ & $\mathrm{x}$ & & & $\mathrm{x}$ & $\mathrm{x}$ & & $\mathrm{x}$ \\
\hline Polycentropodidae & $\mathrm{x}$ & $\mathrm{x}$ & $\mathrm{x}$ & $\mathrm{x}$ & & & & & $\mathrm{x}$ & $\mathrm{x}$ \\
\hline Sericostomatidae & & & & & & & $\mathrm{x}$ & $\mathrm{x}$ & & \\
\hline Xiphocentronidae & & & & & & & & & & $\mathrm{x}$ \\
\hline RIQUEZA OBSERVADA & 49 & 41 & 46 & 44 & 14 & 3 & 30 & 33 & 17 & 46 \\
\hline
\end{tabular}

$\mathrm{SC}=$ Santa Catarina; PMLP = Parque Municipal da Lagoa do Peri; UCAD = Unidade de Conservação Ambiental Desterro; FR = Folha Remanso; PR = Pedra Remanso; FC = Folha Corredeira; PC = Pedra Corredeira; AR = Areia Remanso; FAR = Folha e Areia Remanso.

As curvas de acumulação de táxons (Figuras 2 e 3 ) evidenciaram que o esforço amostral foi suficiente para registrar a riqueza de insetos apenas para o ambiente lótico, pois houve uma tendência à estabilização da curva. Enquanto, para o ambiente lêntico, a assíntota da curva não foi atingida o que indica incremento na riqueza com aumento do esforço amostral.

Quanto ao uso dos hábitats pelos insetos aquáticos, a riqueza observada diferiu entre os tipos de substratos amostrados (Tabela 2), com riqueza muito menor no substrato arenoso tanto nos riachos quanto no

FIGURA 2: Curva de acumulação de riqueza de famílias de insetos aquáticos para o ambiente lótico da Ilha de Santa Catarina/SC, Brasil.

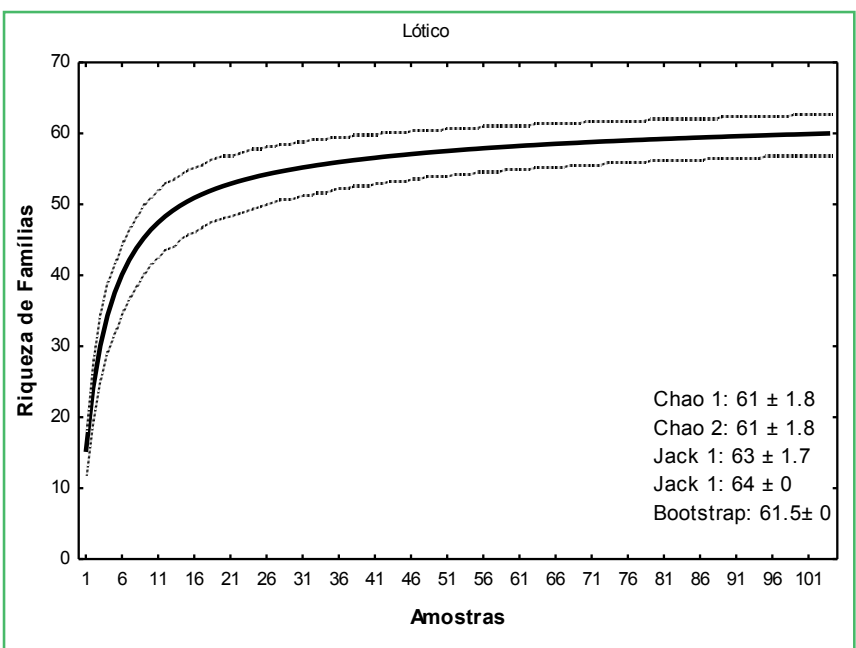

ambiente lêntico. Houve táxons que ocorreram em substratos específicos como: no substrato folha, Dryopidae e Lutrochidae (Coleoptera); em Folha/ Remanso Hydrophilidae (Coleoptera), Corethrelidae (Diptera) e Dicteriadidae (Odonata); no substrato Pedra/Corredeira e Areia/Remanso foram encontradas como táxons exclusivos as famílias Culicidae (Diptera) e Belastomatidae (Hemiptera). As famílias Scirtidae (Coleoptera) e Sericostomatidae (Trichoptera) foram registradas apenas em ambientes de corredeira. Já a família taxonômica Gerridae (Hemiptera) foi apenas observada em áreas de remanso.

FIGURA 3: Curva de acumulação de riqueza de famílias de insetos aquáticos para o ambiente lêntico da Ilha de Santa Catarina/SC, Brasil.

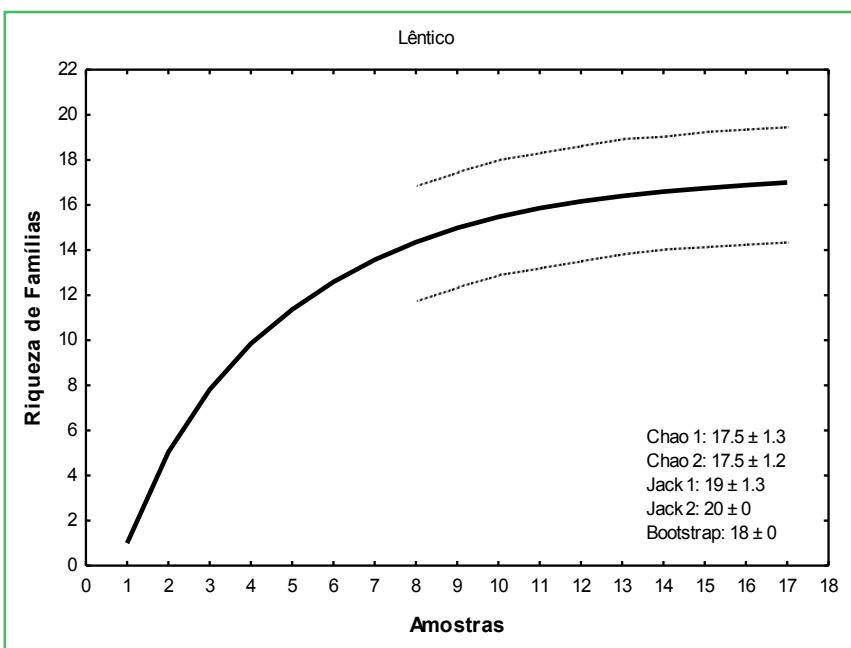


As curvas de rarefação evidenciaram que o substrato areia em trecho de remanso apresentou riqueza menor que os demais habitats (ao comparar uma abundância de 201 indivíduos, Figura 4). A riqueza do substrato pedra em trechos de corredeira e remanso foi maior que o substrato orgânico amostrado (folha) ao comparar a riqueza em uma abundância de 5800 indivíduos (abundância total do hábitat pedra em trecho de remanso; Figura 4).

\section{Discussão}

Os resultados encontrados indicam que a riqueza de insetos aquáticos na Ilha de Santa Catarina se equipara às apresentadas nos trabalhos catarinenses da região continental. A Ilha de Santa Catarina tem sua geologia formada pelo embasamento cristalino e pelos depósitos quaternários de planície costeira sendo uma formação recente, entre 2,588 milhões e 11,5 mil anos atrás (MAACK, 2001). Além da formação recente, a proximidade do continente aliado com a dispersão aérea dos adultos parece explicar a alta diversidade de insetos aquáticos da ilha. Outro fator determinante para encontrar o elevado número de táxons ainda não registrados para o estado foi o grande esforço amostral empregado e amostragem em diversos hábitats e riachos, apesar do esforço amostral dos naturalistas Fritz Muller, Fritz Plaumann e demais pesquisadores brasileiros que contribuíram para o atual conhecimento da entomologia aquática. Como exemplo, o estado catarinense é o terceiro em número de espécies conhecidas da ordem Plecoptera (FROEHLICH, 2011).

Através dos trabalhos ecológicos e de levantamento taxonômico efetuados na região continental do estado foram verificadas 13 famílias de insetos aquáticos sem ocorrência para a região insular. A família Lestidae (Odonata), por exemplo, que não foi registrada na ilha, apresenta apenas dois gêneros com 14 espécies para o Brasil (DE MARCO; VIANNA, 2005) e habitam preferencialmente ambientes lênticos associados às

FIGURA 4: Comparação da riqueza de insetos aquáticos entre os habitats amostrados no sistema lótico entre 2009 e 2012 na Ilha de Santa Catarina/SC, Brasil. As linhas contínuas representam a riqueza média e as linhas tracejadas representam a variação em torno da média $(\mathrm{FR}=$ Folha Remanso; $\mathrm{PR}=$ Pedra Remanso; $\mathrm{FC}=$ Folha Corredeira $\mathrm{PC}=$ Pedra Corredeira; $\mathrm{AR}=\mathrm{Areia}$ Remanso $)$.

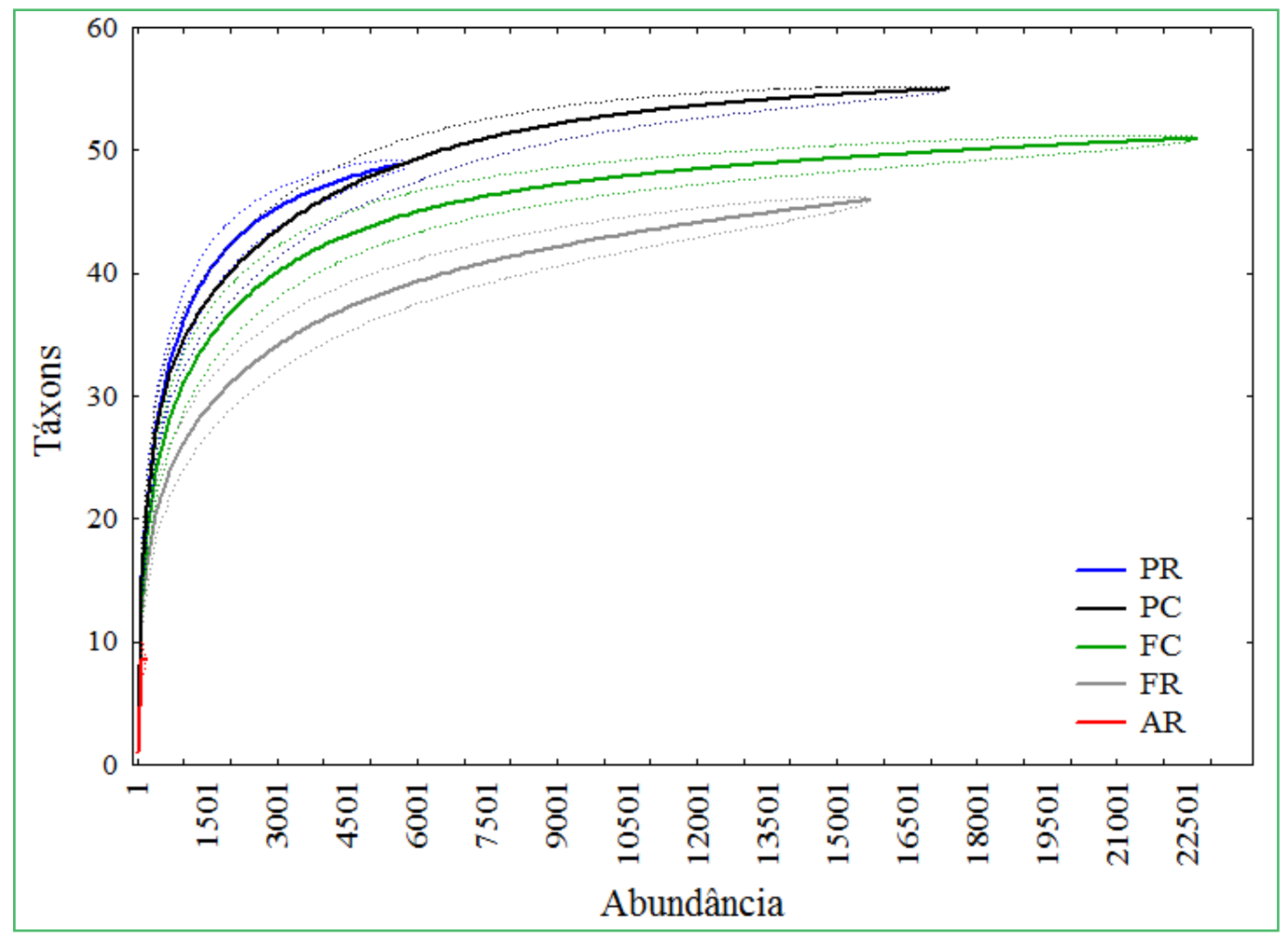


macrófitas (COSTA et al., 2000). Esta família, assim como outras, provavelmente não foi encontrada neste estudo devido à baixa complexidade morfológica das macrófitas na Lagoa Peri com dominância quase exclusiva de Schoenoplectus californicus (C.A. Mey.) Soják na zona marginal (BERLINCK, 1998).

Os ambientes lênticos em geral, tendem a apresentar menor concentração de oxigênio dissolvido na zona profunda e baixa heterogeneidade de substratos quando comparados aos ambientes lóticos, o que sustenta menor diversidade de espécies bentônicas (HAMADA et al., 2014). Dessa forma, embora o esforço amostral tenha sido menor nesse tipo de ambiente em comparação ao ambiente lótico, já era esperada uma menor riqueza nesses locais. Ao comparar a riqueza com outros ambientes lênticos subtropicais, foi observada riqueza similar a de outros lagos naturais e litorâneos de Santa Catarina (TEIVE, 2013), porém menor riqueza que em plantação de arroz irrigado (STERNET et al., 2012) provavelmente reflexo do método de coleta usado e esforço amostral. Neste estudo, apenas uma lagoa foi amostrada, assim um incremento no esforço amostral ao incluir outros ambientes lênticos naturais e artificiais poderia ampliar a ocorrência de outras famílias taxonômicas para a região insular.

O substrato é um fator limitante na distribuição de insetos aquáticos sendo um dos principais fatores para a estruturação da comunidade (BUSS et al., 2004), pois serve de abrigo e alimentação (KIKUCHI; UIEDA, 1998). Em conjunto com o fluxo de água, a colonização do substrato pelos insetos aquáticos é determinada por fatores como a natureza e o tamanho desse substrato. Este é constituído por vários tipos de materiais orgânicos e inorgânicos. Os substratos orgânicos geralmente são formados por folhas de origem alóctone, macrófitas, perifiton ou de origem animal. Os substratos inorgânicos em sua maioria são compostos por areia, cascalho, argila ou ainda matacão (HAMADA et al., 2014). No geral, rochas maiores possuem maior disponibilidade de abrigo, depósito de matéria orgânica nos interstícios, área para crescimento de perifíton e estabilidade frente à correnteza, pois não são facilmente carreados, da mesma forma o acúmulo de folhas no leito dos riachos proporciona uma maior diversidade na fauna devido a maior possibilidade de co-ocorrência de nichos (ALLAN, 1995). No presente trabalho a riqueza foi diferente entre os substratos orgânicos e inorgânicos, sendo que o substrato arenoso teve uma menor riqueza taxonômica, possivelmente por ser um ambiente mais instável (ALLAN, 1995). Em geral, a diversidade aumenta com a estabilidade do substrato e a presença de detritos orgânicos. Nestes é possível encontrar locais onde as exigências metabólicas são menores e os recursos alimentares mais abundantes, propiciando uma maior diversidade de insetos aquáticos (BROOKS et al., 2005), diferentemente do substrato arenoso. Além disso, o efeito agregador do substrato restringe o acesso a este habitat somente pelos insetos escavadores, como Chironomidae (Diptera) (BRUSVEN; PRATHER, 1974).

O estudo representa uma importante contribuição para o conhecimento de insetos aquáticos no estado de Santa Catarina, especialmente para a biodiversidade de ilhas. A Ilha de Santa Catarina apresenta uma alta riqueza taxonômica de insetos aquáticos, porém novos estudos ecológicos e taxonômicos nesta área, bem como no estado catarinense, com uma identificação mais refinada, são necessários e de grande importância para o conhecimento da entomofauna aquática.

\section{Referências}

ALLAN, D. J. Stream ecology: structure and function of running waters. Dordrecht: Kluwer Academic Publishers, 1995. 388 p.

ALVES, J. A. A.; SPRADA, A. T.; GUIMARÃES, T. B. Macroinvertebrates associated to three species of aquatic macrophytes in a lake of Baixada do Maciambú, Parque Estadual da Serra do Tabuleiro, Palhoça, SC. In: CONGRESSO DE ECOLOGIA DO BRASIL, VII, 2006, Caxambú. Anais... Caxambú: SBE. 2006. Versão eletrônica.

BAPTISTA, D. F.; BUSS, D. F.; DROVILlÉ, L. F. M.; NESSIMIAN, J. L. Diversity and habitat preference of aquatic insects along the longitudinal gradient of the Macaé river basin, Rio de Janeiro, Brazil. Revista Brasileira de Biologia, São Carlos, v. 61, n. 2, p. 249-258, 2000.

BATTISTONI, D.; FAVASSA, C. T. A.; TRIQUES, R.; BARP, E. A.; RODRIGUES, G. G. Composição faunística de macroinvertebrados bentônicos ocorrentes na parte baixa do rio jacutinga, Concórdia, SC. Ágora: Revista Divulgação Científica, Mafra, v. 17, n. 1, p. 20-31, 2010.

BEISEL， J. N.; USSEGLIO-POLATERA, P.; THOMAS, S.; MORETEAU, J. C. Stream community structure in relation to 
spatial variation: the influence of mesohabitat characteristics. Hydrobiologia, Brussels, v. 389, p. 73-88, 1998.

BERLINCK, C. N. Levantamento de recursos de vegetação ribeirinha da lagoa do Peri, Ilha de Santa Catarina, SC. Monografia (Bacharelado em Ciências Biológicas) - Universidade Federal de Santa Catarina, Florianópolis. 1998.

BISHEIMER, M. V.; SANTOS, C.; CARLSON, V. E. A mata atlântica da ilha de Santa Catarina. 2 ed. Florianópolis: Lagoa Editora, 2013. 272 p.

BONNET, A; QUEIROZ, M. H. Estratificação vertical de bromélias epifíticas em diferentes estádios sucessionais da Floresta Ombrófila Densa, Ilha de Santa Catarina, Santa Catarina, Brasil. Revista Brasileira de Botânica, São paulo, v. 29, n. 2, p. 217-228, 2006.

BROOKS, A. J.; HAEUSLER, T.; REINFELDS, I.; WILLIAMS, S. Hydraulic microhabitats and the distribution of macroinvertebrate assemblages in riffles. Freshwater Biology, London, v. 50, p. 331344, 2005.

BRUSVEN, M. A.; PRATHER, K. V. Influence of stream sediments on distribution of macrobenthos. Journal of the Entomological Society of British Columbia, Prince George, v. 71, p. 25-32, 1974.

BUSS, D. F.; BAPTISTA, D. F.; NESSIMIAN, J. L.; EGLER, M. Substrate specificity, environmental degradation and disturbance structuring macroinvertebrate assemblages in neotropical streams. Hydrobiologia, Brussels, v. 518, p. 179-188, 2004.

CALlisto, M.; MORETTI, M.; GOULART, M. Macroinvertebrados bentônicos como ferramenta para avaliar a saúde de riachos. Revista Brasileira de Recursos Hídricos, Belo Horizonte, v. 6, n. 1, p. 71-82, 2001.

CARUSO, M. M. L. O desmatamento da Ilha de Santa Catarina de 1500 aos dias atuais. Florianópolis: Editora da UFSC, 1990. $160 \mathrm{p}$.

COLWELL, R. K.; CODDINGTON, J. A. Estimating terrestrial biodiversity through extrapolation. Philosophical Transactions of the Royal Society of London. Series B: Biological Sciences, London, v. 345, n. 1311, p. 101-118, 1994.

COSTA, J. M.; MACHADO, A. B. M.; LENCIONI, F. A. A., SANTOS, T. C. Diversidade e distribuição dos Odonata (Insecta) no estado de São Paulo, Brasil: Parte I - Lista das espécies e registros bibliográficos. Publicações Avulsas do Museu Nacional, Rio de Janeiro, v. 80, p. 1-27, 2000.

DE MARCO, P.; VIANNA, D. M. Distribuição do esforço de coleta de Odonata no Brasil - subsidies para escolha de áreas prioritárias para levantamentos faunísticos. Lundiana, Belo Horizonte, v. 6, p. 13-26, 2005.

DIAS, A. S.; MOLOZZI, J.; PINHEIRO, A. Distribution and occurrence of benthic macroinvertebrates in rivers with rice culture area in Itajaí Valley - SC. Holos Environment, Rio Claro, v. 9, n. 1, p. 45, 2009.

DISNER, G. R.; ROSANELLI, M. L.; SCHÄFER, A. M.; ZANELLA, M. S.; ROSSI, E. M.; ROZA-GOMES, M. F. Avaliação microbiológica da água e de macroinvertebrados bentônicos do rio Guamerim, município de São Miguel do Oeste, SC. Unoesc \& Ciência, Joaçaba, v. 3, n. 1, p. 27-36, 2011.

DOMÍNGUEZ, E.; FERNÁNDEZ, H. R. Macroinvertebrados bentónicos sudamericanos: sistemática y biología. Tucumán: Fundación Miguell Lillo, 2009. 656 p.
FROEHLICH, C. G. Fritz Muller e os insetos aquáticos. Ciência e Cultura, São Paulo, v. 18, n. 4, p. 384-389, 1966.

FROEHLICH, C. G. Checklist of Plecoptera from São Paulo State, Brazil. Biota Neotropica, v. 11, n. 1, 2011. Disponível em: <http://www. biotaneotropica.org.br/v11n1a/en/ abstract?inventory+bn0291101a2011>. Acesso em: 7 ago. 2014.

GALDEAN, N.; CALLISTO, M.; BARBOSA, F. A. R.; ROCHA, L. A. Lotic ecosystems of Serra do Cipó, southeast Brazil: water quality and a tentativa classification based on the benthic macroinvertebrate community. Journal Aquatic Ecosystem Health and Management, Philadelphia, v. 3, p. 545-552, 2000.

GOTELLI, N. J; COLWELL, R. K. Quantifying biodiversity: procedures and pitfalls in the measurement and comparison of species richness. Ecology Letter, Malden, v. 4, n. 4, p. 379-391, 2001.

HAMADA, N.; NESSIMIAN, J. L.; QUERINO, R. B. Insetos aquáticos na Amazônia brasileira: taxonomia, biologia e ecologia. Manaus: Editora do INPA, 2014. 724 p.

HAMMER, O.; HARPER, D. A. T.; RYAN, P. D. PAST: Paleontological statistics software package for education and data analysis. Palaeontologia Eletronica, v.4, n.1, 2001. 9pp.

HENNEMANN, M. C.; PETRUCIO, M. M. Spatial and temporal dynamic of trophic relevant parameters in a subtropical coastal lagoon in Brazil. Environmental Monitoring Assessment, New York, v. 181, p. 347-361, 2011.

HEPP, L. U.; KONIG, R.; TREVISAN, A.; RESTELLO, R. M.; DIAS, A.; MOLOZZI, J.; PINHEIRO, A. Impacto da ocupação urbana sobre a qualidade de águas superficiais: avaliação com indicadores biológicos. In: ENCONTRO NACIONAL DE ÁGUAS URBANA, VI, 2005, Belo Horizonte. Anais..., v. 1, Belo Horizonte: ABRH, 2005. Versão eletrônica.

KALLER, M. D.; HARTMAN, K. J. Evidence of a threshold level of fine sediment accumulation for altering benthic macroinvertebrate communities. Hydrobiologia, Brussels, v. 518, p. 95-104, 2004.

KIKUCHI, R. M.; UIEDA, V. S. Composição da comunidade de invertebrados de um ambiente lótico tropical e sua variação espacial e temporal. In: NESSIMIAN, J. L.; CARVALHO, A. L. (Ed.). Ecologia de insetos aquáticos. Series Oecologia Brasiliensis. Rio de Janeiro: PPGE-UFRJ, 1998. p 157-174.

LISBOA L. K., LEMES, A. L. S., SIEGLOCH, A. L., GONÇALVES-JÚNIOR, J. F, PETRUCIO, M. M.. Temporal dynamics of allochthonous coarse particulate organic matter in a subtropical Atlantic Rain Forest Brazilian stream. Marine and Freshwater Research, Victoria, v. 66, n. 3, 2015.

LISBOA, L. K.; LEMES, A. L. S.; PETRUCIO, M. M. Aquatic invertebrate's distribution in a freshwater coastal lagoon of southern Brazil in relation to water and sediment characteristics. Acta Limnologica Brasiliensia, Botucatu, v. 23, n. 2, p. 119-127, 2011.

MAACK, R. Breves notícias sobre a geologia dos estados do Paraná e Santa Catarina. Brazilian Archives of Biology and Technology, Curitiba, vol.jubilee, p. 169-288, 2001.

MELO, A. S. O que ganhamos "confundindo" riqueza de espécies e equitabilidade em um índice de diversidade? Biota Neotropica, Campinas, v. 8, n. 3, p. 021-027, 2008.

MENDES, H. F.; PINHO, L. C. Brazilian chironomid home. 2014. Disponível em: <https://sites.google.com/site/ brazilianchironomids/home>. Acesso em: 11 set. 2014. 
MOLOZZI, J.; HEPP, L. U.; DIAS, A. S. Influence of rice crop on the benthic community in Itajaí Valley (Santa Catarina, Brazil). Acta Limnologica Brasiliensia, Botucatu, v. 19, n. 4, p. 383-392, 2007.

MUGNAI, R.; NESSIMIAN, J. L.; BAPTISTA, D. F. Manual de identificação de macroinvertebrados aquáticos do estado do Rio de Janeiro. Rio de Janeiro: Technical Books, 2010. 174 p.

NOVAES, M. C.; BISPO, P. C. A new species and notes on Perlidae (Plecoptera) from Paraná and Santa Catarina States, southern Brazil. Zootaxa, Auckland, v. 3765, n. 5, p. 458-468, 2014.

OLIVEIRA, J. S. Análise sedimentar em zonas costeiras: subsídio ao diagnóstico ambiental da Lagoa do Peri - Ilha de Santa Catarina - SC, Brasil. Dissertação (Mestrado em Geografia) - Universidade Federal de Santa Catarina, Florianópolis. 2002.

OLIVEIRA, M.; CALLISTO, A. Benthic macroinvertebrates as bioindicators of water quality in an Atlantic forest fragment. Iheringia, Série Zoologia, Portpo Alegre, v. 100, n. 4, p. 291-300, 2010.

PAPROCKI, H.; HOLZENTHAL, R. W.; BLAHNIK, R. J. Checklist of the Trichoptera (Insecta) of Brazil I. Biota Neotropica, Campinas, v. 4, n. 1, p. 1-22, 2004.

PINTO, A. P.; LAMAS, C. J. E. Navicordulia aemulatrix sp. nov. (Odonata, Corduliidae) from northeastern Santa Catarina State, Brazil. Revista Brasileira de Entomologia, Curitiba, v. 54, n. 4, p. 608-617, 2010.

RAIMUNDI, E. A.; SALLES, F. F.; SOUZA-FRANCO, G. M. Fauna de Leptophlebiidae Banks (Insecta: Ephemeroptera) em fragmentos de Mata Atlântica no oeste de Santa Catarina, Brasil. Biota Neotropica, Campinas, v. 13, n. 4, 2013. Disponível em: <http://www.biotaneotropica.org.br/v13n4/pt/ abstract?article+bn01013042013>. Acesso em: 7 ago. 2014.
RODRIGUES, R. C.; TEIXEIRA, R. A.; CAMPOS, L. A. Comunidade de insetos bentônicos em rio impactado por mineração de carvão em Treviso, Santa Catarina. Tecnologia e Ambiente, Criciúma, v. 13, p. 1-14, 2005.

SALLES F. F.; DA-SILVA E. R.; HUBBARD M. D.; SERRÃO, J. E. As espécies de Ephemeroptera (Insecta) registradas para o Brasil. Biota Neotropica, Campinas, v. 4, n. 2, p. 1-34, 2004.

STENERT, C.; MALTCHIK, L.; ROCHA, O.; Diversity of aquatic invertebrates in rice fields in southern Brazil. Neotropical Biology and Conservation, São Leopoldo, v. 7, n. 1, p. 67-77, 2012.

STERZ, C.; ROZA-GOMES, M. F.; ROSSI, E. M. Análise microbiológica e avaliação de macroinvertebrados bentônicos como bioindicadores da qualidade da água do Riacho Capivara, município de Mondaí, SC. Unoesc \& Ciência, Joaçaba, v. 2, n. 1, p. 7-16, 2011.

TEIVE, L.F. A influência de fatores locais e regionais na dinâmica de estruturação de metacomunidades bênticas de sistemas costeiros do sul do Brasil. 2013. 69 p. Dissertação (Mestrado em Ecologia) - Centro de Ciências Biológicas, Universidade Federal de Santa Catarina, Florianópolis. 2013.

TRIVINHO-STRIXINO, S.; STRIXINO, G. Estrutura da comunidade de insetos aquáticos associados à Pontederia lanceolata Nuttal. Revista Brasileira de Biologia, São carlos, v. 53, p. 103-111, 1993.

TUNDISI, J. G.; MATSUMURA-TUNDISI, T. Limnologia. São Paulo: Oficina de Textos, 2008. 631 p.

VUORI, K-M; JOENSUU, I. Impact of forest drainage on the macroinvertebrates of a small boreal headwater stream: do buffer zones protect lotic biodiversity? Biological Conservation, Boston, v. 77, p. 87-95, 1996. 